\title{
Electrospun gamma-cyclodextrin $(\gamma-C D)$ nanofibers for the entrapment of volatile organic compounds $\uparrow$
}

\author{
Asli Celebioglu ${ }^{\mathrm{ab}}$ and Tamer Uyar*ab
}

Received 3rd September 2013

Accepted 3rd October 2013

DOI: $10.1039 / c 3 r a 44870 c$

www.rsc.org/advances

Electrospinning of gamma cyclodextrin $(\gamma-C D)$ nanofibers from a DMSO-water solvent system was achieved without using a carrier polymeric matrix. The effects of viscosity and rheological properties on the electrospinnability of $\gamma$-CD solutions were examined. XRD and HR-TEM studies confirmed that the electrospun $\gamma$-CD nanofibers were amorphous without showing any particular crystalline packing. The surface area of the $\gamma$-CD nanofibrous web was three times higher than the $\gamma$-CD in powder form. As a preliminary study, we have investigated the molecular entrapment capability of $\gamma$-CD nanofibers. $\gamma$-CD nanofibers were quite successful for entrapping of VOCs (aniline and toluene) by inclusion complexation, whereas $\gamma$-CD in powder form did not show any entrapment capability.

\section{Introduction}

Cyclodextrins (CDs) are cyclic oligosaccharides which are formed naturally as a result of enzymatic degradation of starch. There are three main types of CD; $\alpha$-CD, $\beta$-CD and $\gamma$-CD which are known as native CDs. These native CD are classified according to number of their glucopyranose units and $\alpha$-CD, $\beta$-CD, $\gamma$-CD have six, seven, eight subunits in their molecular structure, respectively., ${ }^{1,2}$ CDs have a toroid-shaped molecular structure with a hydrophobic cavity that can host variety of molecules by forming non-covalent host-guest inclusion complexes (IC) (Fig. 1a)..$^{1-3}$ This intriguing property makes CD molecules quite applicable in various field such as filtration/ separation/purification systems, pharmaceuticals, functional foods, cosmetics, home/personal care and textiles, etc., ${ }^{1,2,4-7}$

CDs are generally used in the form of powder or cross-linked polymeric granules, however, this situation can be a restriction

anstitute of Materials Science \& Nanotechnology, Bilkent University, Ankara, 06800, Turkey.E-mail: tamer@unam.bilkent.edu.tr

${ }^{b}$ UNAM-National Nanotechnology Research Center, Bilkent University, Ankara, 06800, Turkey

$\dagger$ Electronic supplementary information (ESI) available. See DOI: $10.1039 / \mathrm{c} 3 \mathrm{ra} 44870 \mathrm{c}$ during the use of these molecules. ${ }^{1,2,4,5}$ In our pioneer previous studies, we represented a handy solution to this challenge by producing polymer-free nanofibers from CD molecules by using electrospinning method..$^{8-10}$ We obtained CD nanofibers from three different chemically modified cyclodextrins (hydroxypropylbeta-cyclodextrin (HP $\beta \mathrm{CD}$ ), hydroxypropyl-gamma-cyclodextrin (HP $\gamma \mathrm{CD})$ and methyl-beta-cyclodextrin (M $\beta \mathrm{CD}))^{8,9}$ and native CD of $\alpha$-CD and $\beta$-CD. ${ }^{10}$ We have also obtained multifunctional CD nanofibers from their inclusion complex of antibacterial agent (triclosan) ${ }^{11}$ and UV-responsive dye (azobenzene). ${ }^{12}$ We have also achieved green and one-step synthesis of gold nanoparticles incorporated into electrospun $\mathrm{CD}$ nanofibers in which $\mathrm{CD}$ was used as reducing and stabilizing agent as well as fiber template. ${ }^{13}$ Following our studies, few other studies were reported on the electrospinning of modified CD (HP $\beta C D)^{14,15}$ and native CD $(\beta-\mathrm{CD})$ nanofibers. ${ }^{16}$

Among other nanofiber fabrication technique, electrospinning has received great attention due to its simplicity, versatility and cost-effectiveness. ${ }^{17,18}$ Electrospun nanofibers represent extremely high surface area, nano-porous features, very light weight, design flexibility for specific physical and chemical functionalization. ${ }^{17,18}$ All these attractive properties make electrospun nanofibers favourable candidate for numerous applications such as membranes/filters, biotechnology, textiles, sensors, energy, electronics, environment, etc. ${ }^{17-20}$

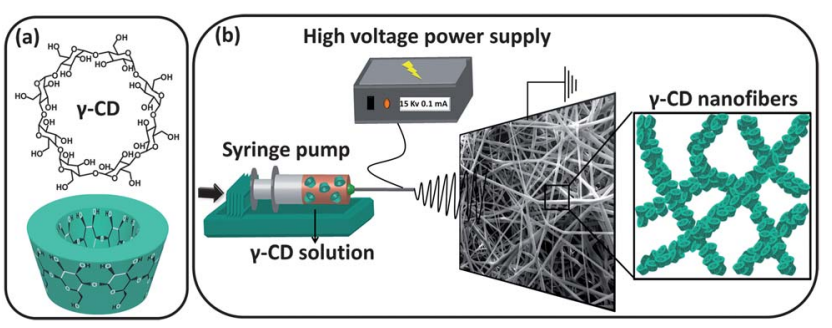

Fig. 1 (a) Chemical structure and the schematic representation of $\gamma$-CD molecule. (b) Schematic representation of the electrospinning of $\gamma$-CD nanofibers. 
Generally speaking, electrospinning has a focus on the use of high molecular weight polymers and high solution concentrations to ensure the entanglements and overlapping between the polymer chains that have crucial importance for producing beadfree and uniform nanofibers. ${ }^{\mathbf{2 1 , 2 2}}$ So, the electrospinning of nonpolymeric systems is much more challenging than the polymeric systems. Yet, few studies have been reported recently on electrospinning of fibers from low molecular weight molecules such as phospholipids, ${ }^{23}$ diphenylalanine, ${ }^{24}$ gemini surfactant, ${ }^{25}$ heteroditopic monomer, ${ }^{26}$ and cyclodextrins. ${ }^{8-16}$ The electrospinning of nanofibers from non-polymeric supramolecular systems is rather new and attractive and therefore, designing and constructing of advanced nanofibrous materials require further investigation and studies. Especially, CDs are one of the most studied supramolecular systems and therefore the electrospinning of these molecules would be quite attractive due to their non-covalent host-guest inclusion complexation capability.

CD molecules can self-assembly and form substantial aggregates in their concentrated solutions via intermolecular hydrogen bonding. ${ }^{27,28}$ The existence of the considerable aggregates makes it possible for the electrospinning of CD nanofibers from their highly concentrated solutions. Hence, we successfully achieved the electrospinning of nanofibers from chemically modified CDs (HP $\beta C D, H P \gamma C D, M \beta C D)$ which have high solubility for the preparation of highly concentrated electrospinning solutions. ${ }^{\mathbf{8 9}}$ In a later study, we were also successful for electrospinning of polymer-free nanofibers from two native $\mathrm{CD} ; \alpha$-CD and $\beta$-CD. ${ }^{10}$ Native CDs $(\alpha-C D, \beta-C D$ and $\gamma$-CD) have limited solubility because of intramolecular hydrogen bonding within the CD molecule which limits the formation of hydrogen bonds with surrounding water molecules. ${ }^{27,29}$ So the electrospinning of native $\mathrm{CD}$ is much more challenging than the chemically modified CD. Yet, we were able to obtain highly concentrated homogeneous solutions of $\alpha$-CD and $\beta-\mathrm{CD}$ by using $10 \%(\mathrm{w} / \mathrm{v}) \mathrm{NaOH}$ aqueous solvent system and we have electrospun nanofibers from $\alpha$-CD and $\beta$-CD. Unfortunately, this solvent system was not suitable for $\gamma$-CD since the required level of concentration could not be reached due to the precipitation of $\gamma$-CD. Electrospinning of $\beta$-CD nanofibers from ionic liquid system was also reported very recently by Ahn et al. ${ }^{\mathbf{1 6}}$ However, to the best of our knowledge, electrospinning of $\gamma$-CD nanofibers and their practical applications as a filtering material for the removal of volatile organic compounds has not been reported yet.

In this study, we found out that dimethyl sulfoxide (DMSO)water $(50 / 50$ ratio, v/v) solvent mixture was a proper solvent system for the preparation of the highly concentrated solution of $\gamma$-CD. Then, electrospinning of $\gamma$-CD nanofibers was successfully achieved. The rheological properties of the $\gamma$-CD solution, morphological and structural characterizations of $\gamma$-CD nanofibers were performed. Moreover, we carried out the BET analyses to investigate and compare the surface areas of $\gamma$-CD powder and $\gamma$-CD nanofibers. As a preliminary study, we have also studied the molecular entrapment capability of $\gamma$-CD nanofibers by capturing the toxic volatile organic molecules (e.g. aniline and toluene) from the surrounding and compared with its powder form.

\section{Results and discussion}

\section{Electrospinning of $\gamma$-CD nanofibers}

The electrospinning solutions were obtained by dissolving $\gamma$-CD in DMSO-water (50/50 ratio, v/v) solvent mixture at the concentration range of $120 \%(\mathrm{w} / \mathrm{v})$ up to $140 \%(\mathrm{w} / \mathrm{v})$. Fig. $1 \mathrm{~b}$ displays the schematic view of the electrospinning of $\gamma$-CD nanofibers. Fig. 2a-c show the representative SEM images of the electrospinning results which were obtained from different concentrations of $\gamma$-CD $(120-140 \%(w / v))$. The fiber diameter distribution of electrospun nanofibers produced from $140 \%$ $(w / v) \gamma$-CD concentration was given in Fig. $2 d$. In addition, the TEM and high resolution (HR-TEM) images of $\gamma$-CD nanofiber were depicted in Fig. 2e and f. Table 1 summarizes the characteristics of the $\gamma$-CD solutions for different concentrations, the morphology and the average fiber diameters of the electrospun $\gamma$-CD nanofibers.

In our previous studies, we achieved the electrospinning of nanofibers from chemically modified CDs (HP $\beta C D, H P \gamma C D$ and $\mathrm{M} \beta \mathrm{CD})$ and native $\mathrm{CDs}(\alpha-\mathrm{CD}$ and $\beta-\mathrm{CD}){ }^{8,9}$ We observed that the electrospinning of CD was very similar to polymeric systems. The solvent type, solution concentration and viscosity and, solution conductivity were the key factors for the electrospinning of CD
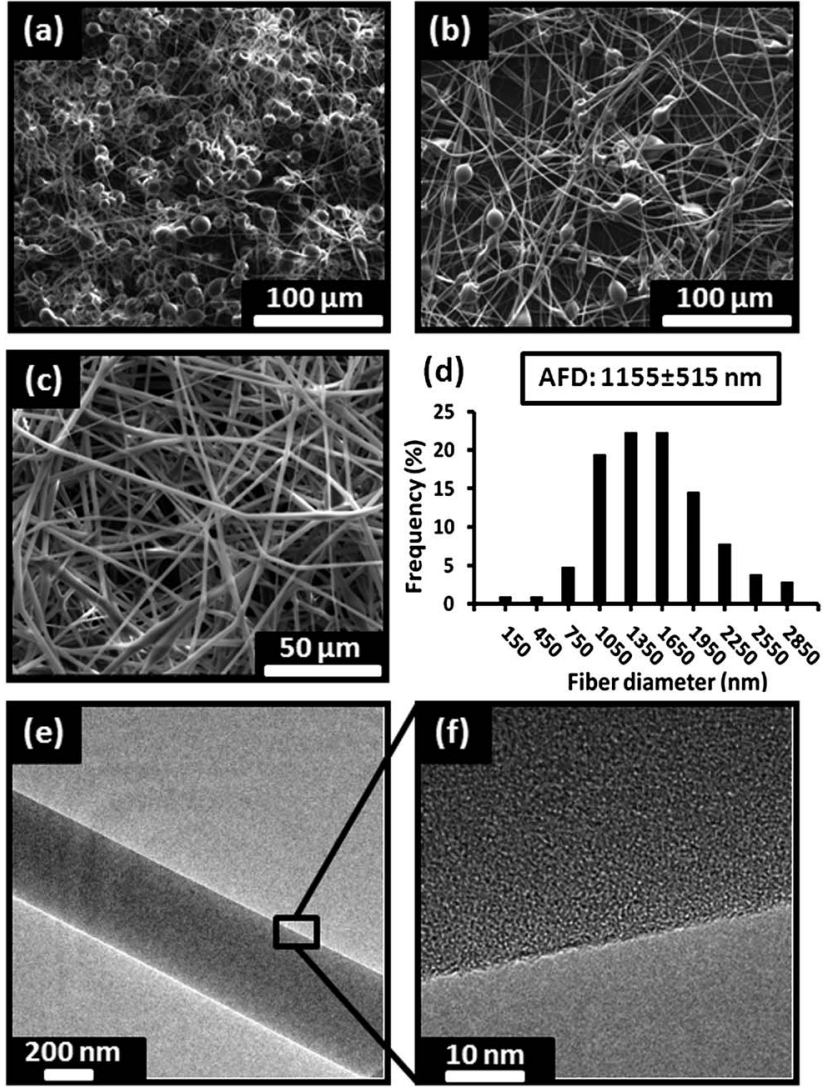

Fig. 2 The representative SEM images of $\gamma$-CD nanofibers obtained from DMSO-water (50/50 ratio, v/v) solvent mixture at (a) 120\% (w/v), (b) 130\% (w/v), (c) $140 \%(w / v) \gamma$-CD concentrations and (d) fiber diameter distribution of electrospun nanofibers produced from $140 \%(\mathrm{w} / \mathrm{v}) \gamma$-CD concentration. (e) TEM and (f) HR-TEM images of electrospun $\gamma$-CD nanofibers (140\% (w/v)). 
Table 1 The characteristics of $\gamma$-CD solutions, average fiber diameter, fiber diameter range and fiber morphology of the electrospun $\gamma$-CD nanofibers

\begin{tabular}{lllll}
\hline Solutions & $\begin{array}{l}\text { Viscosity } \\
(\mathrm{Pa} \mathrm{s})\end{array}$ & $\begin{array}{l}\text { Conductivity } \\
\left(\mu \mathrm{S} \mathrm{cm}^{-1}\right)\end{array}$ & $\begin{array}{l}\text { Average fiber diameter } \\
\text { (fiber diameter range) }(\mathrm{nm})\end{array}$ & Fiber morphology \\
\hline $120 \% \gamma-\mathrm{CD}$ & 0.55 & 2.23 & - & Bead structures \\
$130 \% \gamma$-CD & 0.78 & 2.20 & - & Beaded nano and micro fibers \\
$140 \% \gamma-\mathrm{CD}$ & 1.15 & 1.68 & - & Bead-free nano and micro fibers \\
$140 \% \gamma-\mathrm{CD}+20 \%$ urea & 0.52 & 2.45 & & No fiber formation, only splashes \\
\end{tabular}

nanofibers and all these should be at the optimum level for obtaining uniform and bead-free fiber morphology. For 120\% $(\mathrm{w} / \mathrm{v}) \gamma$-CD solution, highly beaded structure along with very few nanofiber structures was obtained (Fig. 2a). When the concentration was increased to $130 \%(\mathrm{w} / \mathrm{v})$, the nanofibers having few beads were observed (Fig. 2b). Finally, the beads were eliminated for $140 \%(\mathrm{w} / \mathrm{v})$ concentration and uniform nanofibers having average fiber diameter (AFD) of $1155 \pm 515 \mathrm{~nm}$ in the range 200$2900 \mathrm{~nm}$ were produced (Fig. $2 \mathrm{c}$ and d). For lower $\gamma$-CD concentration, the bead structures were observed because of the low viscosity and inadequate amount of $\gamma$-CD aggregates that leaded to the destabilization of electrospinning jet during the process. However, as the concentration was increased to $140 \%$ $(\mathrm{w} / \mathrm{v})$, the bead-free nanofibers were obtained owing to sufficient level of solution viscosity and CD aggregates that provided full stretching of jet and uniform fiber formation. As it is known, low concentrated polymer solution also causes beaded fiber structure because of the poor polymer chain entanglements/overlapping and higher polymer concentrations are required for uniform fiber formation. ${ }^{\mathbf{1 7}, \mathbf{1 8 , 3 0}}$ So, the electrospinning of $\gamma$-CD nanofibers showed similarities with polymeric systems. Here, urea was also added to the optimized concentration of $\gamma$-CD $(140 \%$, (w/v)) solution and only splashes were observed instead of fiber formation (Fig. S1 $\dagger$ ). Because urea can interrupts the hydrogen bonds between CD molecules ${ }^{31,32}$ and reduces the intermolecular interactions that ensure the self-association, aggregation and therefore disrupt the fiber formation during the electrospinning.

The shear rate sweep viscosity and frequency sweep oscillation test were performed to investigate the rheological properties of $\gamma$-CD solutions. Fig. 3 displays the viscosity graphs of $\gamma$-CD solutions for different concentrations as a function of shear rate. The viscosities of $\gamma$-CD solutions did not change significantly for the same concentration against the increasing shear rate showing the Newtonian fluid characteristic of CD systems. On the other hand, there is distinctive increase at viscosity values of $\gamma$-CD solutions with the increasing concentrations owing to higher amount of CD aggregates. The viscosity of $\gamma$-CD solution at $120 \%(\mathrm{w} / \mathrm{v})$ increased from $0.55 \mathrm{~Pa}$ s to 1.15 Pa $\mathrm{s}$ for $140 \%(\mathrm{w} / \mathrm{v})$ and it decreased to $0.52 \mathrm{~Pa}$ s by adding $20 \%$ (w/w) urea (with respect to $\mathrm{CD}$ ) to the highest concentration $(140 \%, w / v)$ (Table 1). This originated from the destruction of CD aggregates by urea which leaded to splashes only during electrospinning process. The frequency sweep oscillation measurements were also studied to determine the viscoelastic properties of the $\gamma$-CD solutions. Fig. 4 displays the storage and loss modulus of $\gamma$-CD solutions as a function of frequency. The results show that, the storage modulus of all concentrations is

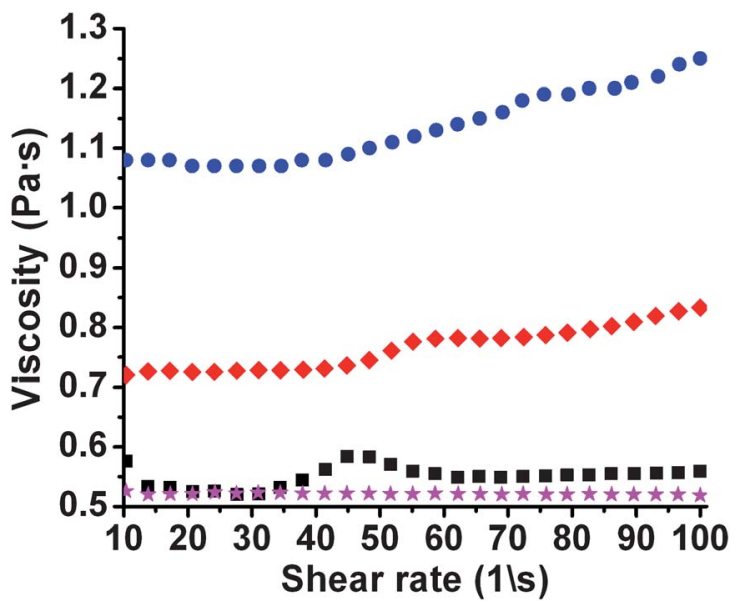

Fig. 3 Viscosity versus shear rate graphs of 120\% ( $\mathbf{\square}), 130 \%(\diamond), 140 \%(\bullet)$, and $20 \%(w / w)$ urea-added $140 \%(w / v)(\star) \gamma$-CD solutions.

higher than their loss modulus values independent from the frequency range. This situation proved that the highly concentrated $\gamma$-CD solutions act as a viscoelastic solid..$^{33}$ For the same $\gamma$-CD concentrations, both storage and loss modulus were stable under the applied frequency range, however, the higher storage and loss modulus values were observed for $140 \%(\mathrm{w} / \mathrm{v})$ $\gamma$-CD solution compared to lower concentrations. The absence of cross-over point between storage and loss modulus also
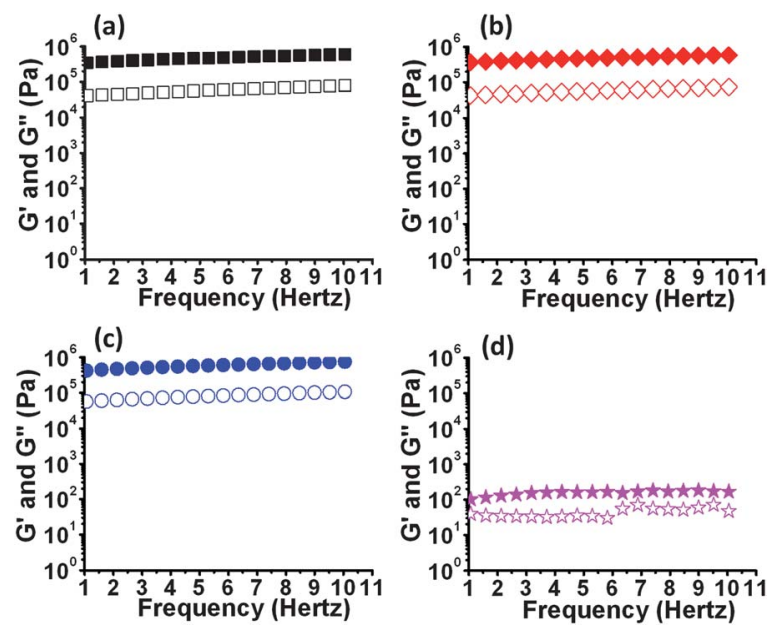

Fig. 4 Frequency depended storage modulus $G^{\prime}$ (filled symbols) and loss

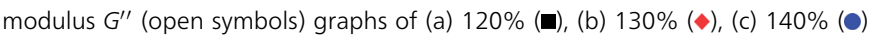
and (d) $20 \%(w / w)$ urea-added $140 \%(w / v)(\star) \gamma$-CD solutions. 
indicates that, $\gamma$-CD solutions show mainly solid-like behavior for all concentrations. The addition of urea in $\gamma$-CD solution also affected the oscillation test results and we observed significant decrease at the modulus values depending on the depression of solid density (Fig. 4d). As a result of urea addition, it was proved that, the presence of considerable amount of $\gamma$-CD aggregates via hydrogen bonding have an important role for the electrospinning of uniform nanofibers from $\gamma$-CD solutions.

\section{Structural characterization of $\boldsymbol{\gamma}$-CD nanofibers}

The native $\mathrm{CD}(\alpha-\mathrm{CD}, \beta$-CD and $\gamma$-CD) have crystalline structures known as cage or channel type. ${ }^{34}$ For instance, in cage-type packing each CD molecules are blocked by neighboring molecules. In the case of channel-type packing, the CD molecules are aligned and stacked on top of each other forming long cylindrical channels. The inclusion complexation with guest molecules commonly results in channel-type crystalline structure. ${ }^{34}$ In this study, the structural characterization of $\gamma$-CD nanofibers was carried out by XRD. For comparison, the XRD measurement of $\gamma$-CD powder was also recorded. Fig. 5 shows the XRD graphs of both $\gamma$-CD nanofibers web and powder. While, as-received powder form of $\gamma$-CD has cage-type crystalline structure, the electrospun $\gamma$-CD nanofibers have halo pattern indicating the absence of definite crystal packing. Similarly, electrospun $\alpha$-CD and $\beta$-CD nanofibers obtained from $\mathrm{NaOH}$ aqueous solvent system $^{\mathbf{1 0}}$ and $\beta$-CD nanofibers obtained from ionic liquid system $^{\mathbf{1 6}}$ did not show any crystal packing either.

The HR-TEM confirmed the distribution of CD molecules through the fiber structure without any particular crystalline aggregation (Fig. 2f). We can say that, the amorphous structure of $\gamma$-CD nanofibers can be originated from the rapid evaporation of solvent during the electrospinning that cause very fast and continuous stretching of jet, so $\gamma$-CD molecules were not able to form crystalline packing. In addition, even we performed XRD measurement 1 year later, the crystalline packing was not observed, $\gamma$-CD nanofibers kept its amorphous structure.

Furthermore, BET analysis was performed to investigate the surface area of $\gamma$-CD powder and $\gamma$-CD nanofibers, comparatively. The multipoint BET surface area for $\gamma$-CD powder was calculated as $1.46 \mathrm{~m}^{2} \mathrm{~g}^{-1}$ which correlates with

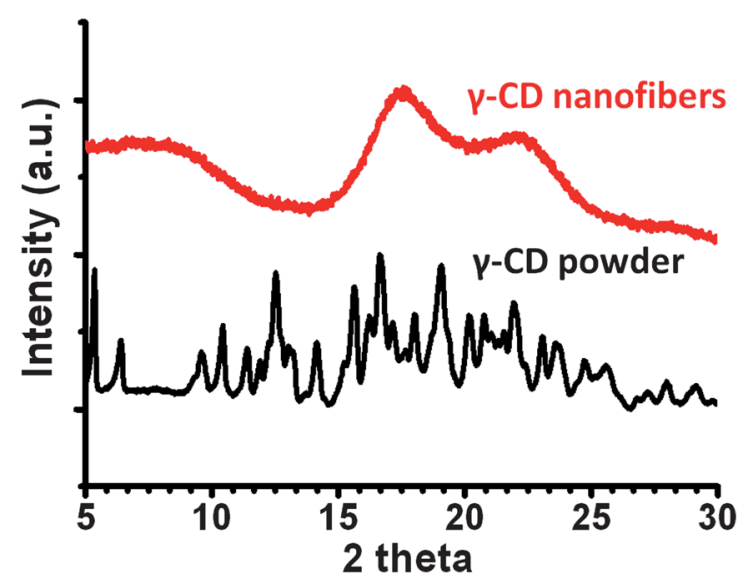

Fig. 5 XRD patterns of as-received $\gamma$-CD powder and $\gamma$-CD nanofibers. the literature report. ${ }^{35,36}$ On the other hand, the surface area of $\gamma$-CD nanofibers was determined as $4.38 \mathrm{~m}^{2} \mathrm{~g}^{-1}$ and this proved the increase of surface area as a result of electrospinning process. So, electrospinning of $\gamma$-CD nanofibers can be considered as an advantage for obtaining high surface area which can benefit the filtration application of these supramolecular structures.

\section{Entrapment of organic vapors by $\gamma$-CD nanofibrous web}

CD can form non-covalent host-guest inclusion complexes with variety of organic molecules and it has been shown that CD can be quite effective for the removal of hazardous organic molecules by inclusion complexation. ${ }^{5}$ In our very recent studies, we have also shown that electrospun polymeric nanofibers functionalized with CD can be potentially used as molecular filters for the removal of organic molecules from the solution ${ }^{37-39}$ or vapor phase ${ }^{40}$ due to the very high surface area of the nanofibers which significantly enhances the complexation efficiency of CD present on the fiber surface. In the present study, the molecular filtration capability of electrospun $\gamma$-CD nanofibrous web was tested by the entrapment of organic waste vapors (aniline and toluene) from the surrounding by inclusion complexation. Here, $\gamma$-CD nanofibrous web was placed in a desiccator saturated with aniline or toluene vapour. For a comparison study, the as-received $\gamma$-CD powder was also placed in the same environment. The analyses of $\gamma$-CD nanofibrous web after exposure to organic vapors were performed by ${ }^{1} \mathrm{H}-\mathrm{NMR}$ (Fig. S2 $\dagger$ ). We observed that $\gamma$-CD nanofibers were quite effective for entrapping aniline and toluene; on the contrary, the $\gamma$-CD powder form could not entrap these molecules from the surrounding. The as-received $\gamma$-CD powder was in cage-type packing in which each CD molecules are blocked by neighboring molecules and therefore CD cavity could not be available for the complexation with aniline and toluene. However, in the case of $\gamma$-CD nanofibers, CD molecules were in amorphous phase without forming any particular crystalline packing structure. Therefore, it is likely that the cavity of $\gamma$-CD was accessible for aniline and toluene molecules for the complexation. Moreover, $\gamma$-CD nanofibrous web has higher surface area compared to $\gamma$-CD powder as proven by BET analysis. With the higher surface area of $\gamma$-CD nanofibrous web, the contact points of CD molecules are higher and they become more available to entrap vapors of organic molecules from the environment. From NMR study, the molar ratio of $\gamma$-CD to aniline and toluene was calculated about $1: 1$ and $1: 0.7$, respectively (Fig. S2 $\dagger$ ). This suggest that the entrapping efficiency of $\gamma$-CD nanofiber match for the aniline was better than the toluene which may be due to the better size match between $\gamma$-CD cavity and guest molecule. ${ }^{41}$ As mention earlier, generally $\gamma$-CD adopt channel-type packing when crystallize from its solution of inclusion complex with guest molecules. ${ }^{34,41}$ After the entrapment experiment, we analyzed $\gamma$-CD nanofibers by XRD and it was observed that $\gamma$-CD nanofibers still kept their amorphous nature even after the inclusion of aniline and toluene. In brief, our preliminary findings suggest that these electrospun CD nanofibers can be very promising candidates for functional filtering nanomaterials and can be 
used as molecular filters and/or nanofilters for the removal of organic volatile compounds (VOCs) from the environment.

\section{Conclusions}

Here, the electrospinning of $\gamma$-CD nanofibers were successfully achieved from DMSO-water $(50 / 50, \mathrm{v} / \mathrm{v})$ solvent system without using any carrier polymeric matrix. The electrospinnability of $\gamma$-CD by itself was due to the existence of $\gamma$-CD aggregates via hydrogen bonding and very high solution viscosity and viscoelastic solid-like behavior of $\gamma$-CD solution in DMSO-water system. The as-received $\gamma$-CD powder is a crystalline material; however, the electrospun $\gamma$-CD nanofibers resulted in amorphous material as confirmed by XRD and HR-TEM studies. BET analysis showed that, the surface area of $\gamma$-CD nanofibrous web was three times higher than $\gamma$-CD powder form. As a preliminary study, electrospun $\gamma$-CD nanofibers were tested for the entrapment of the VOCs (aniline and toluene) from the surrounding. It was found that $\gamma$-CD nanofibers were quite successful for entrapping aniline and toluene by inclusion complexation whereas $\gamma$-CD in powder form did not show any entrapment capability. This suggest that electrospun CD nanofibers can be very promising candidates as filtering material for the removal of organic waste vapors from the environment due to their very high surface area along with their inclusion complexation capability.

\section{Acknowledgements}

State Planning Organization (DPT) of Turkey is acknowledged for the support of UNAM-National Nanotechnology Research Center. The Scientific and Technological Research Council of Turkey (TUBITAK) and EU FP7-PEOPLE-2009-RG Marie CurieIRG (NANOWEB, PIRG06-GA-2009-256428) and The Turkish Academy of Sciences - Outstanding Young Scientists Award Program (TUBA-GEBIP) for funding the research. A. Celebioglu acknowledges TUBITAK-BIDEB for the national graduate study scholarship.

\section{Notes and references}

1 J. Szejtli, Chem. Rev., 1998, 98, 1743.

2 A. R. Hedges, Chem. Rev., 1998, 98, 2035.

3 L. X. Song, L. Bai, X. M. Xu, J. He and S. Z. Pan, Coord. Chem. Rev., 2009, 253, 1276.

4 E. M. M. Del Valle, Process Biochem., 2004, 39, 1033.

5 N. Morin-Crini and G. Crini, Prog. Polym. Sci., 2013, 38, 344. 6 L. B. Devia and A. B. Mandal, RSC Adv., 2013, 3, 5238.

7 W. Song, J. Hu, Y. Zhao, D. Shao and J. Lib, RSC Adv., 2013, 3, 9514.

8 A. Celebioglu and T. Uyar, Chem. Commun., 2010, 46, 6903. 9 A. Celebioglu and T. Uyar, Nanoscale, 2012, 4, 621.

10 A. Celebioglu and T. Uyar, J. Colloid Interface Sci., 2013, 404, 1. 11 A. Celebioglu and T. Uyar, Langmuir, 2011, 27, 6218.

12 M. Chen, S. R. Nielsen, T. Uyar, S. Zhang, A. Zafar, J. Song,

M. Dong and F. Besenbacher, J. Mater. Chem. C, 2013, 1, 850. 13 A. Celebioglu and T. Uyar, RSC Adv., 2013, 3, 10197.
14 J. L. Manasco, C. D. Saquing, C. Tang and S. A. Khan, RSC Adv., 2012, 2, 3778.

15 W. Zhang, M. Chen, B. Zha and G. Diao, Phys. Chem. Chem. Phys., 2012, 14, 9729.

16 Y. Ahn, Y. Kang, M. Ku, Y.-H. Yang, S. Jung and H. Kim, RSC Adv., 2013, 3, 14983.

17 S. Ramakrishna, K. Fujihara, W. Teo, T. Lim and Z. Ma, An Introduction to Electrospinning and Nanofibers, World Scientific Publishing Company, 2005.

18 J. H. Wendorff, S. Agarwal and A. Greiner, Electrospinning: Materials, Processing, and Applications, Wiley-VCH, Germany, 2012.

19 A. Greiner and J. H. Wendorff, Angew. Chem., Int. Ed., 2007, 46, 5670.

20 R. Sahay, P. Suresh Kumar, R. Sridhar, J. Sundaramurthy, J. Venugopal, S. G. Mhaisalkar and S. Ramakrishna, J. Mater. Chem., 2012, 22, 12953.

21 P. Gupta, C. Elkins, T. E. Long and G. L. Wilkes, Polymer, 2005, 46, 4799.

22 S. L. Shenoy, W. D. Bates, H. L. Frisch and G. E. Wnek, Polymer, 2005, 46, 3372.

23 M. G. McKee, J. M. Layman, M. P. Cashion and T. E. Long, Science, 2006, 311, 353.

24 G. Singh, A. M. Bittner, S. Loscher, N. Malinowski and K. Kern, Adv. Mater., 2008, 20, 2332.

25 M. P. Cashion, X. Li, Y. Geng, M. T. Hunley and T. E. Long, Langmuir, 2010, 26, 678.

26 X. Yan, M. Zhou, J. Chen, X. Chi, S. Dong, M. Zhang, X. Ding, Y. Yu, S. Shaod and F. Huang, Chem. Commun., 2011, 47, 7086.

27 M. Messner, S. Kurkov, P. Jansook and T. Loftsson, Int. J. Pharm., 2010, 387, 199.

28 M. Bonini, S. Rossi, G. Karlsson, M. Almgren, P. Nostro and P. Baglioni, Langmuir, 2006, 22, 1478.

29 J. Szejtli, J. Mater. Chem., 1997, 7, 575.

30 T. Uyar and F. Besenbacher, Polymer, 2008, 49, 5336.

31 L. Szente, J. Szejtli and G. Kis, J. Pharm. Sci., 1998, 87, 778.

32 W. Hinze, D. Pharr, Z. Fu and W. Burkert, Anal. Chem., 1989, 61, 422.

33 O. Jazkewitsch and H. Ritter, Macromolecules, 2011, 44, 375. 34 W. Saenger, J. Jacob, K. Gessler, T. Steiner, D. Hoffmann, H. Sanbe, K. Koizumi, S. M. Smith and T. Takaha, Chem. Rev., 1998, 98, 1787.

35 R. Guo and L. D. Wilson, J. Appl. Polym. Sci., 2012, 125, 1841. 36 J.-F. Ju, M.-J. Syu, H.-S. Teng, S.-K. Chou and Y.-S. Chang, Sens. Actuators, B, 2008, 132, 319.

37 T. Uyar, R. Havelund, J. Hacaloglu, F. Besenbacher and P. Kingshott, ACS Nano, 2010, 4, 121.

38 F. Kayaci, Z. Aytac and T. Uyar, J. Hazard. Mater., 2013, 261, 286.

39 T. Uyar, R. Havelund, Y. Nur, J. Hacaloglu, F. Besenbacher and P. Kingshott, J. Membr. Sci., 2009, 332, 129.

40 T. Uyar, R. Havelund, Y. Nur, A. Balan, J. Hacaloglu, L. Toppare, F. Besenbacher and P. Kingshott, J. Membr. Sci., 2010, 365, 409.

41 T. Uyar, M. A. Hunt, H. S. Gracz and A. E. Tonelli, Cryst. Growth Des., 2006, 6, 1113. 